\title{
El LUgar de la Psicología en las inVestigaciones empíricas del SUICIDIO EN ARGENTINA: UN ESTUDIO BIBLIOMÉTRICO
}

\author{
State of psychological empirical RESEARCh on suicide in ARgentine: \\ A BIBLIOMETRIC STUDY
}

\author{
Pablo Ezequiel Flores Kanter* \\ *Licenciado en Psicología. Investigador Part-Time en la Secretaría de Investigación de la Universidad Siglo 21. \\ E-Mail: pablo.floreskanter@ues21.edu.ar \\ Leonor de Tejeda 1866, Barrio Juniors - (5000) Córdoba, Argentina.
}

\section{RESUMEN}

El suicidio constituye un problema de salud pública que tiene un gran impacto económico, social y psicológico a nivel mundial. Cada año se estima que mueren cerca de un millón de personas en todo el mundo debido al suicidio. De lo anterior se desprende la importancia de contar con investigaciones empíricas que sustenten todo tipo de accionar en la problemática del suicidio, desde políticas en salud pública hasta tratamientos psicológicos. Dada la relevancia del tema, el estudio bibliométrico realizado tuvo como principal objetivo verificar la producción empírica sobre el suicidio en Argentina, haciendo especial énfasis en el lugar de la Psicología en dicha producción. Sintetizando, los resultados permiten concluir que la producción empírica sobre el suicidio en los principales países de Sudamérica es muy baja; más aún si se considera solamente a Argentina. Otra conclusión importante tiene que ver con la menor implicancia de la ciencia psicológica en el abordaje empírico del suicidio, comparando los aportes desde un enfoque médico. Estas conclusiones invitan a reflexionar sobre la necesidad de un mayor abordaje empírico del suicidio en la región, y particularmente en Argentina.

Palabras clave: Argentina; Estudio bibliométrico; Psicología basada en la evidencia; Suicidio; Investigaciones; Sudamérica.

\section{ABSTRACT}

Suicide is a major problem of public health leading to great economic, social, and psychological costs worldwide. Around one million people die every year on the world due to suicide. Furthermore, suicide is responsible for half of violent deaths worldwide exceeding even the combination of deaths due to war or homicide. Taking into account the above, researchers around the world recommended concerted efforts to generate actions, research, and investigation with the objective of reduce risk to suicide. Therefore, evidence from empirical research supporting public health policies and psychological treatments is needed. The present article introduces a bibliometric study aimed to examine the state of empirical research on suicide in Argentina, and specially the amount of psychological empirical research. Specifically, research methodology consists of the following aspects: As regards the criteria of inclusion / exclusion of articles, the search focused on the detection of empirical suicide articles. In this sense, the main criterion for inclusion in the analysis was based on studies that have been published or were being published in scientific journals. For the aforementioned reasons, they were excluded from this study theoretical article, books, book chapters, theses, and conference presentation. Also, those articles that used the term suicide to address problems outside the addressed (e.g., Suicide Gene Therapy) were ex- 
cluded from the analysis. In relation to the search strategy, the main database used has been Scopus, which maintains a leading position among bibliographic databases. In this sense, the journals included in the database have very high quality standards. The two principal terms used in the search have been suicide and suicidal. The selection of these two words is because account for the main variables involved in suicide, as expressed in terms such Suicide attempt, Suicidal act, Suicidal behavior and Suicide ideation. The full search term applied in SCOPUS is transcribed below: TITLE (suicide) OR TITLE (suicidal) AND NOT TITLE (suicidal gene) AND NOT TITLE (gene therapy) AND NOT TITLE (suicidal dna) AND NOT TITLE (suicide dna) AND NOT TITLE (suicide drugs) AND NOT TITLE (eryptosis) AND NOT TITLE (streptococcus pneumoniae) AND NOT TITLE (assisted suicidal) AND NOT TITLE (assisted suicide) AND NOT TITLE (iron suicide) AND NOT TITLE (proton suicide method) AND LIMIT-TO (DOCTYPE, “ar") OR LIMIT-TO (DOCTYPE, "re") OR LIMIT-TO (DOCTYPE, "ip"). Other databases consulted were LILACS and PUBMED. PUBMED is open access and is the main database of health sciences in the world, produced by the National Library of Medicine in the United States. These bases were mainly used to supplement the data obtained by SCOPUS and specifically, were used to achieve a broader and more complete picture of empirical studies on suicide, carried out specifically in Argentina. LILACS search term used was suici\$ (Argentina). In PUBMED search term used was suici* (Argentina). The sign * and $\$$ were used to retrieve words of the same family, such as suicide or suicidal. Finally, for the calculation of bibliometric indicators we took into account the suggestions given in the international literature. Thus, the following descriptive data were analyzed: number of items by country, number of items by country depending on the year, international collaboration of, considering year too, language of publication of articles, and thematic areas involved. Results show that scientific literature concerning suicide in South America is limited, even more in Argentine. Moreover, most research in suicide is based on medical rather than psychological approach. Indeed, regarding the number of publications $(f=443)$ in South American countries, they only represent $1.49 \%$ of the ar- ticles published worldwide. The scientific research on suicide is even more critical in Argentina. Specifically, when considering publications on Scopus, LILACS and PUBMED, empirical research in this country represents only $1.41 \%$ of the extant literature on suicide. However, in recent years there have been an increasing number of publications from which may reflects a burgeoning interest in this topic. Results also indicate a greater collaboration between Argentina and other countries, particularly United States, as indicated by coauthorship studies $(f=6)$. Surprisingly, collaboration with (main producer of scientific articles in the South American region) is scarce $(f=2)$. Finally, take into account publications from in research databases analyzed (Scopus, LILACS, and PUBMED) we note that most research on suicide has been published in medical journals $(f=30$; $71.42 \%$ ), while a lower number of articles has been published in psychological journals $(f=5 ; 1.19 \%)$. Globally, these results claim for more empirical research in suicide from psychologist, particularly in Argentina.

Key words: Argentina; Bibliometric study; Evidence-based Psychology; Suicide; Researchs; South America.

\section{INTRODUCCIÓN}

El suicidio puede definirse como la muerte causada por una conducta auto-infligida dañina, que tuvo además un carácter intencional; esto es, la persona se infringió un daño con el fin de causar su propia muerte y ha fallecido a causa de dicho acto (Wenzel, Brown \& Beck, 2009). El suicidio constituye además un problema de salud pública que tiene un gran impacto económico, social y psicológico a nivel mundial (Ministerio de Salud de la Nación Argentina, 2014; Wasserman, D. \& Wasserman, C., 2009).

Cada año se estima que mueren cerca de un millón de personas en todo el mundo debido al suicidio (Ellis \& Rutherford, 2008). 
En función de lo anterior, resulta relevante dar cuenta de los datos epidemiológicos que se presentan tanto a nivel mundial como en Argentina. A nivel mundial, en el año 2012 la causa de muerte por suicidio ascendía a $1.4 \%$ en relación al total de muertes registradas. Sin embargo, este porcentaje se incrementa si uno considera el total de muertes por causas intencionales violentas (i.e., violencia interpersonal, conflictos armados y suicidio), donde el suicidio representa el 56\%. Asimismo, la edad aparece como un factor importante a considerar, dado que el suicidio se presenta como la segunda causa principal de muerte en adultos jóvenes de 15 a 29 años. En relación al sexo, los estados miembros de la Organización Mundial de la Salud (OMS) dieron cuenta de una razón de suicidio hombre / mujer de 3.2, o sea es tres coma dos veces más frecuente en hombres (Ministerio de Salud de la Nación Argentina, 2014).

Similar en muchos aspectos a lo observado mundialmente, en Argentina los datos del año 2013 dan cuenta de una tasa de mortalidad por suicidio de 7.4 cada 100.000 habitantes. Si se consideran sus 41.45 millones de habitantes, esta tasa de mortalidad equivale a 3.037 muertes por dicha causa. Sumado a lo anterior, el suicidio representa el $56.5 \%$ de las muertes violentas intencionales registradas en 2013. Es importante notar también aquí que la mayor cantidad de muertes por suicidio se presentó entre los adolescentes y adultos jóvenes (i.e, entre 15 y 24 años), alcanzando un total de 897 muertes (equivalente a un $30 \%$ de las muertes por suicidio), seguido por el grupo de adultos mayores (de 75 años o más). Así, la tasa de suicidio por cada 100.000 habitantes en el grupo etario de 24 años presentó un valor de 12.7 y de 10.6 en el grupo etario de 75 años o más. Asimismo, en el grupo de adolescentes y adultos jóvenes se evidencia una tendencia creciente desde el año 2000 (tasa menor a 10 cada 100.000 habitantes) al 2013 (tasa igual a 12.7 cada 100.000 habitantes); mientras que en el grupo de adultos mayores se presenta una tendencia decreciente desde el año 2000 (tasa igual a 20 cada 100.000 habitantes) al 2013 (tasa igual a 10.6 cada 100.000 habi- tantes). Finalmente, la razón de muertes por suicidio de hombre / mujer fue igual a 4 (4 hombres por cada mujer). En los hombres, las mayores tasas se presentaron en los grupos de 15 a 24 años (tasa igual a 20.4 por 100.000 habitantes) y de 75 años o más (tasa igual a 24.9 por 100.000 habitantes). Mientras que en las mujeres, las mayores tasas se dieron en los grupos de 15 a 24 años (tasa igual a 4.9 por 100.000 habitantes) y de 45 a 54 años (tasa igual a 3.7 por 100.000 habitantes). En función de los datos descritos a nivel mundial y en Argentina, el Ministerio de Salud de la Nación Argentina (2014) afirma que el suicidio constituye un importante problema de salud pública y señala la necesidad de desarrollar líneas de trabajo que posibiliten prevenirlo, especialmente en los grupos de adolescentes / jóvenes y adultos mayores.

Siendo una gran problemática en el ámbito de la salud pública, para Ellis y Rutherford (2008) llama la atención que las investigaciones indiquen que son pocas las intervenciones terapéuticas que actualmente reducen el riesgo suicida. Aún así, la literatura da cuenta de diversos estudios centrados en indagar aquellas variables psicológicas que permiten explicar y predecir el suicidio (Chioqueta \& Stiles, 2007; Ellis \& Rutherford, 2008; Rudd, Trotter \& Williams, 2009; Smith, Alloy \& Abramson, 2006; Wenzel \& Beck, 2008; Wenzel et al., 2009; Williams, Barnhofer, Crane \& Beck, 2005). De lo anterior se desprende la importancia de contar con investigaciones empíricas que sustenten todo tipo de accionar en la problemática del suicidio, desde políticas en salud pública hasta tratamientos psicológicos (Asencio, Jiménez, Santos \& Herrera, 2008).

El principal objetivo del estudio bibliométrico que se informa fue verificar la producción empírica sobre el suicidio en Argentina, poniendo especial énfasis en el lugar de la Psicología en dicha producción. Hasta el momento no se han verificado estudios de este tipo en Sudamérica. Por tal motivo, se pretende no solo informar sobre la producción científica del suicidio evidenciada en Argentina, sino también hacer una comparación con los restantes países de la región. 


\section{MÉTOdO}

\section{CRITERIOS DE INCLUSIÓN / EXCLUSIÓN DE ARTíCULOS}

La búsqueda se centró en la detección de artículos de carácter empírico sobre el suicidio. En este sentido, el principal criterio de inclusión para el análisis se basó en estudios publicados, o que estuvieran en vías de publicación en revistas científicas. Por estos motivos se excluyeron artículos teóricos, libros, capítulos de libros, tesis y ponencias de congresos. También se excluyeron aquellos artículos que si bien hacían uso del término suicidio, el mismo se usaba para referirse a cuestiones ajenas a la problemática abordada (e.g. Suicide Gene Therapy).

\section{ESTRATEGIA DE BÚSQUEDA DE ARTÍCULOS}

La principal base de datos utilizada ha sido SCOPUS, ya que mantiene una posición destacada entre las bases de datos bibliográficas (Andrés, 2009). En este sentido, sus revistas incluidas tienen estándares muy altos de calidad. Además, el sistema de SCOPUS facilita la recuperación de datos de mucha importancia para cualquier estudio bibliométrico.

Los dos términos principales utilizados en la búsqueda han sido suicide y suicidal. La selección de estas dos palabras se debe a que aparecen en las principales variables implicadas en el suicidio (Wenzel et al., 2009), como queda expresado en los términos en inglés suicide attempt, suicidal act, suicidal behaviour y suicide ideation. El término de búsqueda completo aplicado en SCOPUS se transcribe a continuación:

TITLE (suicide) OR TITLE (suicidal) AND NOT TITLE (suicidal gene) AND NOT TITLE (gene therapy) AND NOT TITLE (suicidal dna) AND NOT TITLE (suicide dna) AND NOT TITLE (suicide drugs) AND NOT TITLE (eryptosis) AND NOT TITLE (streptococcus pneumoniae) AND NOT TITLE (assisted suicidal) AND NOT TITLE (assisted suicide) AND NOT TITLE (iron suicide) AND NOT TITLE (proton suicide method) AND LIMIT-TO (DOCTYPE, “ar") OR LIMIT-TO (DOCTYPE, "re") OR LIMITTO (DOCTYPE, “ip”).

Otras bases consultadas fueron LILACS y PUBMED. PUBMED es de acceso abierto y es la principal base de datos de ciencias de la salud en el mundo, producida por la National Library of Medicine de Estados Unidos. Estas bases se utilizaron principalmente para complementar los datos obtenidos mediante SCOPUS y específicamente, fueron usadas para lograr un panorama más amplio y completo de los estudios empíricos sobre el suicidio, realizados específicamente en Argentina.

El término de búsqueda utilizado en LILACS fue suici\$ (Argentina). El signo \$ permite recuperar palabras de una misma familia, como pueden ser suicidio, o suicida por ejemplo. Para PUBMED el término de búsqueda utilizado fue suici* (Argentina). El signo * permite recuperar palabras de una misma familia, como pueden ser suicidio, o suicida por ejemplo.

\section{MÉtodos estadísticos}

Para calcular los indicadores bibliométricos se tuvieron en cuenta las sugerencias dadas en la literatura internacional (Andrés, 2009). En concreto, se analizaron los siguientes datos descriptivos: cantidad de artículos de cada país, cantidad de artículos por país en función del año, colaboración internacional de Argentina, colaboración internacional de Argentina considerando el año, idioma de publicación de los artículos y áreas temáticas implicadas.

Finalmente, para el análisis de los datos se utilizaron los softwares Open Office Base, Open Office Calc, Kettle-Spoon y Excel.

\section{Resultados}

En primer lugar se desarrollan los resultados obtenidos con SCOPUS.

El criterio de búsqueda utilizado arrojó un total de 29.626 estudios a nivel mundial. Dada la cantidad de países involucrados, en la Tabla 
1 solo se muestran los primeros 15 países, siendo la mayor cantidad de publicaciones las de Estados Unidos $(f=9.274)$, Reino Unido $(f=2.032)$, Alemania $(f=1.334)$, Canadá $(f=1.308)$ y Australia $(f=1.164)$.

En la Tabla 2 se presenta el total de publicaciones con sus porcentajes correspondientes, evidenciadas en los principales países sudamericanos. En la región, los que tienen mayor cantidad de publicaciones sobre el tema son Brasil $(f=302)$, Colombia $(f=60)$, Chile $(f=52)$ y Argentina $(f=17)$.

Con respecto a las publicaciones en Argentina en los últimos 7 años, la cantidad de estudios ha ido variando. Se observan dos artículos publicados en 2009, tres en el 2010, uno solo tanto en el 2011 como en el 2012 y 2013 y tres artículos en el año 2015.

Por otra parte, es importante contemplar también la colaboración internacional que han evidenciado las publicaciones sobre el suicidio en Argentina. La mayor colaboración se ha hecho con Estados Unidos $(f=6)$, mientras que las restantes se distribuyen con Brasil $(f=2)$, Hungría $(f=1)$ y Alemania $(f=1)$.

Por último, en la Tabla 3 se presentan las áreas temáticas de las revistas, en las que han sido publicados los artículos evidenciados en Argentina. Los anteriores resultados permiten visualizar que Medicina $(f=9 ; 52.94 \%)$ es el área principal de especialización de las revistas, seguida de Psicología $(f=3 ; 17.65 \%)$.

Finalmente se describen los resultados obtenidos a través de LILACS y PUBMED.

En LILACS se encontraron 11 artículos de Argentina. La menor parte, publicada en los últimos 7 años (sólo tres artículos: uno en 2011, uno en 2013 y otro en 2014). Además, ninguno de ellos informa colaboración internacional. Por último y similar a lo que se encontró en SCOPUS, las principales áreas temáticas de las revistas implicadas son Medicina $(f=7 ; 64 \%)$, seguida de Psicología $(f=2 ; 18 \%)$ y las revistas multidisciplinarias $(f=2 ; 18 \%)$.

Por su parte, en PUBMED se encontraron 14 publicaciones en Argentina. Al igual que con LILACS, la menor parte se publicó en los últimos 7 años (42.85\%). Tampoco se encon- tró aquí colaboración internacional. Finalmente, el total de las publicaciones corresponde a revistas de Medicina.

En el Cuadro 1 se presentan los artículos sobre el suicidio publicados en los últimos 10 años en Argentina, sus autores, año de publicación, como así también su título.

\section{Discusión}

El desarrollo y la implementación de intervenciones efectivas y basadas en la evidencia es fundamental a la hora de reducir los índices de suicidio o conductas relacionadas con el mismo (Brown \& Jager-Hyman, 2014; Wasserman et al., 2012). Siguiendo esta línea, la investigación realizada se propuso indagar la producción empírica sobre el suicidio en Sudamérica, especialmente en Argentina.

A un nivel más general, los resultados muestran que los principales países de Sudamérica tienen un porcentaje de producción científica sobre el suicidio bastante bajo si se lo compara con lo observado a nivel mundial (ver Tabla 1). El total de publicaciones de los países sudamericanos contemplados $(f=443)$ representa un $1.49 \%$ de los artículos publicados mundialmente.

Al analizar la producción argentina sobre el suicidio el panorama es más crítico. Así, aun considerando el total de publicaciones evidenciadas tanto en SCOPUS como en LILACS y PUBMED, dan cuenta solo del $1.41 \%$ del total de publicaciones sobre el tema a nivel mundial (cabe resaltar que se está considerando como total la cantidad de resultados encontrados en SCOPUS, por lo que el porcentaje obtenido debería ser menor si se considerara un total en conjunto entre todas las bases). Sin embargo, la producción en los últimos años ha ido en aumento dando cuenta tal vez de una tendencia en alza, con respecto al interés por el estudio del suicidio en Argentina.

En relación con la colaboración internacional de Argentina en sus publicaciones, los resultados indican una mayor colaboración con Estados Unidos $(f=6)$, lo cual resulta 
muy positivo si se tiene en cuenta que Estados Unidos es el principal productor de artículos empíricos sobre el suicidio a nivel mundial $(f=9.274 ; 31.31 \%)$. Llama la atención aquí que la colaboración con Brasil (principal productor de artículos científicos de la región sudamericana) se muestre muy pobre (sólo dos artículos han sido encontrados).

Finalmente, una mención especial merece el análisis de las áreas de especialización de las revistas en las cuales se han publicado los artículos, dado que sus áreas temáticas predominantes pueden tomarse como indicador de la implicación de las distintas ciencias, particularmente de la ciencia psicológica, en el estudio del suicidio. Tomando todas las bases de datos (i.e., SCOPUS, LILACS y PUBMED) y centrando el análisis en las publicaciones de Argentina, es posible ver que casi tres cuartas partes de los artículos fueron publicados en revistas de la especialidad médica $(f=30 ; 71.42 \%)$, mientras que un porcentaje mucho más bajo de estos artículos fue publicado en revistas de especialidad psicológica $(f=5 ; 1.19 \%)$.

Sintetizando, los resultados permiten concluir que la producción empírica sobre el suicidio en los principales países de Sudamérica es muy baja; más aún si se considera solamente la región argentina. Otra conclusión importante tiene que ver con la menor implicancia de la ciencia psicológica en el abordaje empírico del suicidio, en comparación con los aportes desde un enfoque médico.

Las anteriores conclusiones invitan a reflexionar sobre la necesidad de un mayor abordaje empírico del suicidio en la región, y particularmente en Argentina. En este sentido, no sólo es importante ser consumidor de li- teratura científica a la hora de abordar alguna problemática de salud pública, como ser el suicidio, sino que es fundamental que se lleven a cabo investigaciones empíricas. Estas últimas deben tener como principal objetivo aportar al avance del conocimiento sobre el suicidio, pero además replicar los hallazgos evidenciados en contextos diferentes (Fabrigar \& Wegener, en prensa; Singh, Ang \& Leong, 2003). Asimismo, en función de la importancia que han demostrado tener las variables psicológicas en la predicción, explicación y tratamiento del suicidio (Ellis \& Rutherford, 2008; O'Connor \& Nock, 2014; Wenzel \& Beck, 2008; Wenzel et al., 2009), la Psicología como ciencia debe lograr un rol más protagónico en el estudio del comportamiento suicida. Por último, resulta importante seguir consolidando la colaboración internacional con países que tienen un mayor desarrollo en cuanto a publicaciones empíricas sobre el suicidio, particularmente entre Argentina y otros países sudamericanos, principalmente Brasil.

La investigación que se informa tuvo como meta principal hacer un llamado de atención a la comunidad científica de Psicología en Argentina con respecto a la importancia de abordar la problemática del suicidio desde un enfoque empírico, y la necesidad imperante de avanzar en el conocimiento de esta problemática mediante el aumento de investigaciones sobre el tema. Sólo el aumento de estudios y el uso adecuado de los resultados obtenidos, podrán lograr que se den respuestas eficaces y eficientes a la problemática del suicidio, tanto en la región en general como en Argentina en particular. 
TABLA 1

TOTALES Y PORCENTAJES DE PUBLICACIONES SEGÚN PAIIS

\begin{tabular}{l|cc|}
\hline \multicolumn{1}{c|}{ País } & $n$ & $\%^{1}$ \\
\hline Estados Unidos & 9274 & \\
Reino Unido & 2032 & 31.31 \\
Alemania & 1334 & 6.86 \\
Canadá & 1308 & 4.50 \\
Australia & 1164 & 4.42 \\
Francia & 829 & 3.93 \\
Suecia & 798 & 2.80 \\
Italia & 711 & 2.69 \\
Japón & 595 & 2.40 \\
España & 494 & 2.01 \\
China & 452 & 1.67 \\
Suiza & 421 & 1.53 \\
Israel & 410 & 1.42 \\
India & 405 & 1.38 \\
Países Bajos & 378 & 1.37 \\
\hline
\end{tabular}

${ }^{1}$ Porcentajes calculados en base al total de publicaciones $(N=29.626)$.

TABLA 2

TOTALES Y PORCENTAJES DE PUBLICACIONES EN LOS PRINCIPALES PAÍSES SUDAMERICANOS

\begin{tabular}{l|cc|}
\hline \multicolumn{1}{|c|}{ País } & $n$ & $\%^{1}$ \\
\hline Brasil & 302 & 1 \\
Colombia & 60 & .2 \\
Chile & 52 & .2 \\
Argentina & 17 & .1 \\
Perú & 4 & .01 \\
Ecuador & 3 & .01 \\
Uruguay & 2 & .01 \\
Venezuela & 1 & .001 \\
Paraguay & 1 & .001 \\
Bolivia & 1 & .001 \\
\hline
\end{tabular}

${ }^{1}$ Porcentajes calculados en base al total de publicaciones $(N=29.626)$. 
TABLA 3

CANTIDAD DE PUBLICACIONES EN REVISTAS ESPECIALIZADAS SEGÚN ÁREA TEMÁTICA

\begin{tabular}{|l|l|}
\hline \multicolumn{1}{|c|}{ Área temática } & $n$ \\
\hline Psicología & 3 \\
Neurociencia & 2 \\
Medicina & 9 \\
Bioética & 1 \\
Multidisciplinaria & 2 \\
\hline Total & 17 \\
\hline
\end{tabular}

CUADRO 1

PUBLICACIONES ARGENTINAS DE LOS ÚLTIMOS 10 AÑOS SOBRE EL SUICIDIO

\begin{tabular}{|c|c|c|}
\hline Autores & Año & Título \\
\hline $\begin{array}{l}\text { Fuchsova, B., Álvarez Juliá, A., Rizavi, H.S., } \\
\text { Frasch, A.C. \& Pandey, G.N. }\end{array}$ & 2015 & $\begin{array}{l}\text { Altered expression of neuroplasticity-related genes in } \\
\text { the brain of depressed suicides. }\end{array}$ \\
\hline $\begin{array}{l}\text { Zvolensky, M.J., Bakhshaie, J., Garza, M., Valdi- } \\
\text { vieso, J., Ortiz, M., Bogiaizian, D., Robles, Z., } \\
\text { Schmidt, N.B. \& Vujanovic, A. }\end{array}$ & 2015 & $\begin{array}{l}\text { The role of anxiety sensitivity in the relation between } \\
\text { experiential avoidance and anxious arousal, depres- } \\
\text { sive, and suicidal symptoms among latinos in primary } \\
\text { care. }\end{array}$ \\
\hline $\begin{array}{l}\text { Rebok, F., Teti, G.L., Fantini, A.P., Cardenas-Delga- } \\
\text { do, C., Rojas, S.M., Derito, M.N.C. \& Daray, F.M. }\end{array}$ & 2015 & $\begin{array}{l}\text { Types of Borderline Personality Disorder (BPD) in } \\
\text { patients admitted for suicide-related behavior. }\end{array}$ \\
\hline Szmulewicz, A.G., Smith, J.M. \& Valerio, M.P. & 2015 & $\begin{array}{l}\text { Suicidality in clozapine-treated patients with schizoph- } \\
\text { renia: role of obsessive-compulsive symptoms. }\end{array}$ \\
\hline Toro-Martínez, E. & 2014 & $\begin{array}{l}\text { Risk and suicide in open door institutions. Liability and } \\
\text { forensic issues. }\end{array}$ \\
\hline Martínez, D.V. & 2014 & $\begin{array}{l}\text { Deliberate self-harm (DSH). Between constraint and } \\
\text { relief. }\end{array}$ \\
\hline
\end{tabular}

Continúa 


\begin{tabular}{|c|c|c|}
\hline Autores & Año & Título \\
\hline Martínez, C. & 2014 & $\begin{array}{l}\text { Provincial public center for crisis intervention and } \\
\text { psycho-social rehabilitation. A path towards communi- } \\
\text { tary suicidology. }\end{array}$ \\
\hline $\begin{array}{l}\text { Teti, G.L., Rebok, F., Grendas, L.N., Rodante, D., } \\
\text { Fógola, A. \& Daray, F.M. }\end{array}$ & 2014 & $\begin{array}{l}\text { Systematic review of risk factors for suicide and sui- } \\
\text { cide attempt among psychiatric patients in Latin Ame- } \\
\text { rica and Caribbean. }\end{array}$ \\
\hline $\begin{array}{l}\text { Teti, G.L., Rebok, F., Rojas, S.M., Grendas, L. \& } \\
\text { Daray, F.M. }\end{array}$ & 2014 & $\begin{array}{l}\text { Pacientes hospitalizadas por ideación e intento de } \\
\text { suicidio en un hospital de salud mental: Característi- } \\
\text { cas clínico-demográficas y seguimiento a seis meses. }\end{array}$ \\
\hline Morra, A.P., Elorza, C. \& Echenique, H. & 2013 & $\begin{array}{l}\text { Perfil epidemiológico de los intentos de suicidio aten- } \\
\text { didos en el Hospital Dr. Lucio Molas, Santa Rosa, La } \\
\text { Pampa, } 2011 .\end{array}$ \\
\hline $\begin{array}{l}\text { Bella, M.E., Acosta, L., Villace, B., De Neira, M.L., } \\
\text { Enders, J. \& Fernández, R. }\end{array}$ & 2013 & $\begin{array}{l}\text { Analysis of mortality from suicide in children, adoles- } \\
\text { cents and youth. Argentina, 2005-2007. }\end{array}$ \\
\hline Bella, M.E. & 2012 & $\begin{array}{l}\text { Risk factors and behaviors among children and ado- } \\
\text { lescents hospitalized for a suicidal attempt. }\end{array}$ \\
\hline Girard, G.A. \& Silber, T.J. & 2011 & $\begin{array}{l}\text { The aftermath of adolescent suicide: Clinical, ethical, } \\
\text { and spiritual issues. }\end{array}$ \\
\hline Sola, M. & 2011 & $\begin{array}{l}\text { Sociodemografía del suicidio en la población adoles- } \\
\text { cente y joven en Argentina. }\end{array}$ \\
\hline $\begin{array}{l}\text { Vázquez, G.H., Gonda, X., Zaratiegui, R., Loren- } \\
\text { zo, L.S., Akiskal, K. \& Akiskal, H.S. }\end{array}$ & 2010 & $\begin{array}{l}\text { Hyperthymic temperament may protect against suici- } \\
\text { dal ideation. }\end{array}$ \\
\hline Marin, G.R. \& Baspineiro, B. & 2010 & $\begin{array}{l}\text { Posictal neurogenic pulmonary edema secondary to } \\
\text { acute poisoning by organochlorine pesticide in an } \\
\text { adolescent suicide attempt. }\end{array}$ \\
\hline Bella, M.E., Fernández, R.A. \& Willington, J.M. & 2010 & $\begin{array}{l}\text { Depression and the conduct disorder are the most fre- } \\
\text { quent pathologies in child and adolescent suicide at- } \\
\text { tempt. }\end{array}$ \\
\hline
\end{tabular}




\begin{tabular}{|c|c|c|}
\hline Autores & Año & Título \\
\hline Bins, H.D.D.C., Doler, C. \& Teitelbaum, P.O. & 2009 & Homicide followed by suicide: Case report. \\
\hline Gaitan, M.I. & 2009 & $\begin{array}{l}\text { A multicentre study on suicide outcomes following } \\
\text { subthalamic stimulation for Parkinson's disease. }\end{array}$ \\
\hline Alba, P., Dominguez, D. \& Schteingart, A. & 2008 & Antidepressants and suicidality: Primum non nocere. \\
\hline $\begin{array}{l}\text { Bella, M.E, Fernández, A.R, Acevedo, G. \& } \\
\text { Willington, J.M. }\end{array}$ & 2008 & $\begin{array}{l}\text { Socio-demographic and psychopathological analysis } \\
\text { of child and adolescent attempted suicides. }\end{array}$ \\
\hline Outomuro, D. & 2007 & Morality of suicide in dementia. \\
\hline Casullo, M.M. \& Fernández Liporace, M. & 2007 & $\begin{array}{l}\text { Estudio comparativo sobre adolescentes en riesgo } \\
\text { suicida. }\end{array}$ \\
\hline $\begin{array}{l}\text { Matusevich, D., Ruíz, M., Vairo, M.C., Job, A. \& } \\
\text { Finkelsztein, C. }\end{array}$ & 2006 & $\begin{array}{l}\text { Tentativas de suicidio en adolescencia con psicofár- } \\
\text { macos de los padres: Estudio descriptivo. }\end{array}$ \\
\hline Toro Martínez, E. \& Rudelir, M. & 2005 & Assessment of suicide risk: Clinical and legal aspects. \\
\hline
\end{tabular}

Nota: Los artículos se encuentran ordenados según el año de publicación. 


\section{REFERENCIAS BIBLIOGRÁFICAS}

Andrés, A. (2009). Measuring academic research. How to undertake a bibliometric study. Cambridge: Chandos Publishing.

Alba, P., Domínguez, D. \& Schteingart, A. (2008). Antidepressants and suicidality: Primum non nocere. Vertex, 19 (82), 357-63.

Asencio, J.M.M., Jiménez, E.G., Santos, F.J.M. \& Herrera, J.C.M. (2008). Salud pública basada en la evidencia. Recursos sobre la efectividad de intervenciones en la comunidad [Evidence based public health. Resources on efectiveness of community interventions]. Revista Española de Salud Pública, 82(1), 5-20.

Bella, M.E., Acosta, L., Villace, B., De Neira, M.L., Enders, J. \& Fernández, R. (2013). Analysis of mortality from suicide in children, adolescents and youth. Argentina, 2005-2007. Archivos Argentinos de Pediatria, 111(1), 16-21. http://dx. doi.org/10.1590/S0325-00752013000100005.

Bella, M.E. (2012). Risk factors and behaviors among children and adolescents hospitalized for a suicidal attempt. Revista Médica de Chile, 140(11), 1417-1424. http://dx.doi.org/10.4067/ S0034-98872012001100006.

Bella, M.E., Fernández, R.A. \& Willington, J.M. (2010). Depression and the conduct disorder are the most frequent pathologies in child and adolescent suicide attempt. Archivos Argentinos de Pediatría, 108(2), 124-129. http://dx.doi.or g/10.1590/S0325-00752010000200006.

Bella, M.E., Fernández, A.R., Acevedo, G. \& Willington, J.M. (2008). Socio-demographic and psychopathological analysis of child and adolescent attempted suicides. Vertex, 19(78), 10-16.

Bins, H.D.D.C., Doler, C. \& Teitelbaum, P.O. (2009). Homicide followed by suicide: Case report. Revista de Psiquiatria do Rio Grande do Sul, 31(2), 128-131. http://dx.doi.org/10.1590/ S0101-1082009000200008

Brown, G.K. \& Jager-Hyman, S. (2014). Evidencebased psychotherapies for suicide prevention.
Future directions. American Journal of Preventive Medicine, 47(32), 186-194. http://dx.doi.or g/10.1016/j.amepre.2014.06.008

Casullo, M.M., Fernández Liporace, M. \& González, N.C. (2007). Estudio comparativo sobre adolescentes en riesgo suicida [Suicide risk among adolescent students: A comparative study]. Biblioteca Institucional CAICYT "Ricardo A. Gietz”. Recuperado el 12 de julio de 2016 de http://www.caicyt-conicet.gov.ar/bibli o/items/show/38969.

Chioqueta, A.P. \& Stiles, T.C. (2007). Dimensions of the Dysfunctional Attitude Scale (DAS-A) and the Automatic Thoughts Questionnaire (ATQ-30) as cognitive vulnerability factors in the development of suicide ideation. Behavioural and Cognitive Psychotherapy, 35, 579589.

Ellis, T.E. \& Rutherford, B. (2008). Cognition and suicide: Two decades of progress. International Journal of Cognitive Therapy, 1(1), 47-68. http://dx.doi.org/10.1521/ijct.2008.1.1.47

Fabrigar, L.R. \& Wegener, D.T. (En prensa). Conceptualizing and evaluating the replication of research results. Journal of Experimental Social Psychology.

Fuchsova, B., Álvarez Juliá, A., Rizavi, H.S., Frasch, A.C. \& Pandey, G.N. (2015). Altered expression of neuroplasticity-related genes in the brain of depressed suicides. Neuroscience, 299(03), 1-17. http://dx.doi.org/10.1016/j.neu roscience. 2015.04 .057

Gaitan, M.I. (2009). A multicentre study on suicide outcomes following subthalamic stimulation for Parkinson's disease. Brain, 131(10), 27202728. http://dx.doi.org/10.1093/brain/awn214.

Girard, G.A. \& Silber, T.J. (2011). The aftermath of adolescent suicide: Clinical, ethical, and spiritual issues. Adolescent Medicine State of the Art Review, 22(2), 229-239.

Marin, G.R. \& Baspineiro, B. (2010). Posictal neurogenic pulmonary edema secondary to acute poisoning by organochlorine pesticide in an adolescent suicide attempt. Archivos Argentinos 
de Pediatría 108(2), 28-32. http://dx.doi.org/ 10.1590/S0325-00752010000200013

Martínez, C. (2014). Provincial public center for crisis intervention and psycho-social rehabilitation. A path towards communitary suicidology. Vertex, 25(115), 195-202.

Martínez, D.V. (2014). Deliberate self-harm (DS H). Between constraint and relief. Vertex, 25 (115), 213-219.

Matusevich, D., Ruiz, M., Vairo, M.C., Job, A. \& Finkelsztein, C. (2006). Tentativas de suicidio en adolescencia con psicofármacos de los padres: Estudio descriptivo [Attempted suicide in adolescents with psychotropic drugs parent: Descriptive study]. Vertex, 17(70), 446-451.

Ministerio de Salud de la Nación Argentina. (2014). Mortalidad por suicidio en Argentina 2000-2013 (Informe técnico preliminar) [Suicide mortality in Argentina 2000-2013 (Preliminary technical report)]. Dirección de Estadísticas e Información de Salud, Serie 5(57).

Morra, A.P., Elorza, C. \& Echenique, H. (2013). Perfil epidemiológico de los intentos de suicidio atendidos en el Hospital Dr. Lucio Molas, Santa Rosa, La Pampa, 2011 [Epidemiological profile of suicide attempts treated at the Hospital Dr. Lucio Molas, Santa Rosa, La Pampa, 2011]. Revista Argentina de Salud Pública, 4(15), 2631.

O'Connor, R.C. \& Nock, M.K. (2014). The psychology of suicidal behaviour. Lancet Psychiatry, 1, 73-85. http://dx.doi.org/10.1016/ S22150366(14)70222-6

Outomuro, D. (2007). Morality of suicide in dementia. The American Journal of Bioethics, 7 (6), 64-65. http://dx.doi.org/10.1080/1526516 0701347577

Rebok, F., Teti, G.L., Fantini, A.P., Cárdenas-Delgado, C., Rojas, S.M., Derito, M.N.C. \& Daray, F.M. (2015). Types of Borderline Personality Disorder (BPD) in patients admitted for suiciderelated behavior. Psychiatric Quarterly, 86(1), 49-60. http://dx.doi.org/10.1007/s11126-0149317-3
Rudd, M.D., Trotter, D.R.M. \& Williams, B. (2009). Psychological theories of suicidal behaviour. En D. Wasserman \& C. Wasserman (Eds.), Suicidology and suicide prevention. A global perspective (pp. 159-165). New York: Oxford University Press.

Singh, K., Ang, S.H. \& Leong, S.M. (2003). Increasing replication for knowledge accumulation in strategy research. Journal of Management, 29(4), 533-549. http://dx.doi.org/10.101 6/S0149-2063(03)00024-2

Smith, J.M., Alloy, L.M. \& Abramson, L.Y. (2006). Cognitive vulnerability to depression, rumination, hopelessness, and suicidal ideation: Multiple pathways to self-injurious thinking. Suicide and Life-Threatening Behavior, 36(4), 443-445.

Sola, M. (2011). Sociodemografía del suicidio en la población adolescente y joven en Argentina [Sociodemographics of suicide among adolescents and young adults in Argentina]. Salud(i) Ciencia, 21, 639-641.

Szmulewicz, A.G., Smith, J.M. \& Valerio, M.P. (2015). Suicidality in clozapine-treated patients with schizophrenia: Role of obsessive-compulsive symptoms. Psychiatry Research, 230(1), 50-55. http://dx.doi.org/10.1016/j.psy chres.20 15.07 .089

Teti, G.L., Rebok, F., Grendas, L.N., Rodante, D., Fógola, A. \& Daray, F.M. (2014). Pacientes hospitalizadas por ideación e intento de suicidio en un hospital de salud mental: Características clínico-demográficas y seguimiento a seis meses [Patients hospitalized for suicidal ideation and suicide attempt in a mental health hospital: Clinico-emographical features and six months follow-up]. Dossier, 25(115).

Teti, G.L., Rebok, F., Rojas, S.M., Grendas, L. \& Daray, F.M. (2014). Systematic review of risk factors for suicide and suicide attempt among psychiatric patients in Latin America and Caribbean. Revista Panamericana de Salud Pública, 36(2), 124-133. 
Toro Martínez, E. \& Rudelir, M. (2005). Assessment of suicide risk: clinical and legal aspects. Vertex, 16(61), 196-205.

Toro Martínez, E. (2014). Risk and suicide in open door institutions. Liability and forensic issues. Vertex, 25(115), 220-224.

Vazquez, G.H., Gonda, X., Zaratiegui, R., Lorenzo, L.S., Akiskal, K. \& Akiskal, H.S. (2010). Hyperthymic temperament may protect against suicidal ideation. Journal of Affective Disorder, 127(1-3), 38-42. http://dx.doi.org/10.1016/j. jad.2010.04.015.

Wasserman, D. \& Wasserman, C. (Eds.). (2009). Suicidology and suicide prevention. A global perspective. New York: Oxford University Press.

Wasserman, D., Rihmer, Z., Rujescu, D., Sarchiapone, M., Sokolowski, M., Titelman, D., Zalsman, G., Zemishlany, Z. \& Carli, V. (2012). The European Psychiatric Association (EPA) guidance on suicide treatment and prevention. European Psychiatry, 27, 129-141. http://dx. doi.org /10.1016/j.eurpsy.2011.06.003

Wenzel, A. \& Beck, A.T. (2008). A cognitive model of suicidal behavior: Theory and treatment.
Applied and Preventive Psychology, 12, 189201. http://dx.doi.org/10.1016/j.appsy.2008.05. 001

Wenzel, A., Brown, G.K. \& Beck, A.T. (2009). Cognitive for suicida therapy patients. Scientific and clinical applications. Washington, DC: American Psychological Association.

Williams, J.M.G., Barnhofer, T., Crane, C. \& Beck, A.T. (2005). Problem solving deteriorates following mood challenge in formerly depressed patients with a history of suicidal ideation. Journal of Abnormal Psychology, 114(3), 421431. http://dx.doi.org/10.1037/0021-843X.11 4.3.421

Zvolensky, M.J., Bakhshaie, J., Garza, M., Valdivieso, J., Ortiz, M., Bogiaizian, D., Robles, Z., Schmidt, N.B. \& Vujanovic, A. (2015). The role of anxiety sensitivity in the relation between experiential avoidance and anxious arousal, depressive, and suicidal symptoms among latinos in primary care. Cognitive Therapy and Research, 39, 688-696. http:// dx.doi.org/10. 1007/s10608-015-9696-2

Universidad Siglo 21

Córdoba - República Argentina

Fecha de recepción: 17 de septiembre de 2015

Fecha de aceptación: 10 de agosto de 2016 